\title{
GLYCEROLIPID BIOSYNTHESIS IN PEROXISOMES VIA THE ACYL DIHYDROXYACETONE PHOSPHATE PATHWAY*
}

\author{
Amiya K. Hajra and James E. Bishop \\ Department of Biological Chemistry and \\ Mental Health Research Institute \\ University of Michigan \\ Ann Arbor, Michigan 48109
}

In recent years, acyl dihydroxyacetone phosphate [acyl-DHAP†] has been shown to be a precursor of glycerolipids and glycerol-ether lipids. ${ }^{1.2}$ Acyl-DHAP was discovered as a rapidly labeled lipid that was formed in crude mitochondrial fraction from ${ }^{32} \mathrm{Pi}$ or $\gamma={ }^{32} \mathrm{P}[\mathrm{ATP}]{ }^{3}$ This rapid labeling was due to the enzymatic dephosphorylation and rephosphorylation of endogenous acyl-DHAP present in the crude mitochondrial fraction (equation 1):

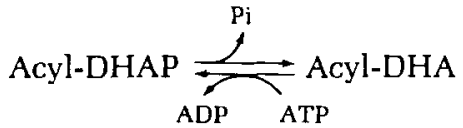

Acyl-DHAP was later shown to be biosynthesized by a direct acylation of DHAP with acyl-CoA, catalyzed by DHAP acyltransferase (EC 2.3.1.42) (equation 2):

$$
\text { Acyl-CoA }+\mathrm{DHAP} \rightleftharpoons \mathrm{Acyl}-\mathrm{DHAP}+\mathrm{CoA}
$$

Subsequently it was shown that acyl-DHAP was enzymatically reduced by NADPH (1-acyl, G-3-P:NADP ${ }^{+}$oxidoreductase EC 1.1.1.101) to 1-acyl-sn-glycerol-3-phosphate or lysophosphatidate ${ }^{5,6}$ (equation 3):

$$
\text { Acyl-DHAP + NADPH } \rightarrow \text { 1-acyl-sn-G-3-P + NADP }{ }^{+}
$$

A further enzymatic acylation converts lysophosphatidate to phosphatidate: ${ }^{1,2}$

$$
\text { 1-acyl,G-3-P + acyl-CoA } \rightarrow \text { 1,2 diacyl G-3-P }
$$

This set of above reactions (equations 2, 3 , and 4) provides an alternate pathway, i.e., other than diacylation of G-3-P $\mathrm{P}^{1}$, for the biosynthesis of phosphatidic acid which is shown to be the precursor of all glycerides and phosphoglycerides $^{2.7}$ (Fic. 1).

Acyl-DHAP is also shown to be the precursor of glycerol lipids containing an ether bond (glycerol-ether lipids and plasmalogens). Acyl-DHAP reacts with long-chain alcohols to form 1-O-alkyl dihydroxyacetone-3-phosphate (alkylDHAP]. ${ }^{8-10}$

$$
\text { Acyl-DHAP }+ \text { ROH } \rightarrow \text { Alkyl-DHAP }+ \text { Fatty Acid }
$$

*This work is supported by National Institute of Health Grants NS 08841 and NS 15747.

† Abbreviations used: DHAP - dihydroxyacetone phosphate; DHAPAT-dihydroxyacetone phosphate acyltransferase; GP. sn-glycerol-3-phosphate; GPAT-glycerophosphate acyltransferase. 
Long-chain alcohols are formed by enzymatic reduction of acyl-CoA's with NADPH. ${ }^{11}$ Alkyl DHAP is enzymatically reduced and acylated by reactions analogous to (3) and (4) above to form the ether analog of phosphatidic acid. ${ }^{1}$ This phosphatidate analog is then converted to 1-alkyl, 2-acyl-sn-glycerol-3-phosphoethanolamine which, as described by different workers, is dehydrogenated to form ethanolamine plasmalogens. ${ }^{9,12}$ The reactions involving acyl-DHAP and its conversion to different glycerolipids along with the other reactions for glycerolipid biosynthesis are summarized in FIGURE 1.

The relative importance of acyl-DHAP pathway, compared to that of the glycerol-3-P pathway (FIG. 1) has been a subject of study for a number of years. It has been established that glycerol ether lipids in higher organisms are biosynthesized solely via acyl-DHAP pathway. ${ }^{1.13}$ However, the contribution of the acylDHAP pathway for the biosynthesis of nonether glycerolipid is still not firmly established, though a number of workers have indirectly shown that in a variety of systems a major amount of glycerolipid is synthesized via acyl-DHAP. ${ }^{13-15}$

The key enzymes of the acyl-DHAP pathway, i.e., DHAPAT, alkyl-DHAP synthase and acyl/alkyl DHAP:NADPH oxidoreductase have been shown to be widely distributed in different mammalian tissues. ${ }^{6.16 .17}$ When initially checked, these enzymes were shown to be present in a membrane-bound form in both the mitochondrial and microsomal fractions of different tissues. ${ }^{6.16}$ However, the distribution was somewhat unusual in that, in liver and kidney, these enzymes were found to be enriched in the mitochondrial fraction whereas in other tissues such as brain, lung, testis, and adipose tissue, these enzymes appeared to be localized in the microsomal fraction. This anomalous distribution prompted us to reinvestigate the subcellular distribution of these enzymes. A careful subcellular fractionation of guinea pig liver according to the procedure of de Duve et al. ${ }^{18}$ and assay of different enzymes in these fractions revealed that DHAPAT is enriched neither in mitochondria nor in microsomes but in an intermediate "light mitochondrial" fraction which mainly contains lysosomes and peroxisomes. ${ }^{19}$ Comparison with different subcellular marker enzymes showed that the distribution pattern of DHAPAT was very similar to the distribution of catalase and uric acid oxidase, rather than to lysosomal marker enzymes, thus indicating that this enzyme is probably localized in peroxisomes. ${ }^{19}$ DHAPAT was also found to be similarly enriched in the light mitochondrial fraction of rat liver. ${ }^{20}$

To establish firmly that DHAPAT is indeed localized in peroxisomes, it was necessary to isolate peroxisomes free of lysosomal contamination. For this purpose the method developed by Leighton et al. ${ }^{21}$ was followed. Rats were injected intraperitoneally with Triton-WR 1339; and after 3-5 days the liver subcellular fractions were isolated. The distribution pattern of DHAPAT and various marker enzymes was found to be very similar to that of normal rats. ${ }^{20}$ The light mitochondrial fraction obtained from these rats was subjected to linear sucrose density gradient (density 1.05 to $1.25 \mathrm{~g} / \mathrm{ml}$ ) centrifugation. The results showed that DHAPAT followed the pattern of the peroxisomal marker enzymes, uric acid oxidase and catalase (high density] rather than the lysosomal marker enzyme, acid phosphatase (low density). ${ }^{20}$ These results established that most of DHAPAT is indeed localized in peroxisomes. A small amount [5-10\%] of DHAPAT in rat liver seemed to be present in the microsomes.

The other key enzymes of the acyl-DHAP pathway, i.e., acyl/alkyl DHAP:NADPH oxidoreductase and alkyl-DHAP synthase also seemed to be enriched in the peroxisomal fraction ${ }^{22}$ (FIG. 2). However, a large fraction of acyl-DHAP oxidoreductase was also found to be present in the microsomal fraction. ${ }^{20}$ The alkyl-DHAP synthase of rat liver seemed to have a similar 


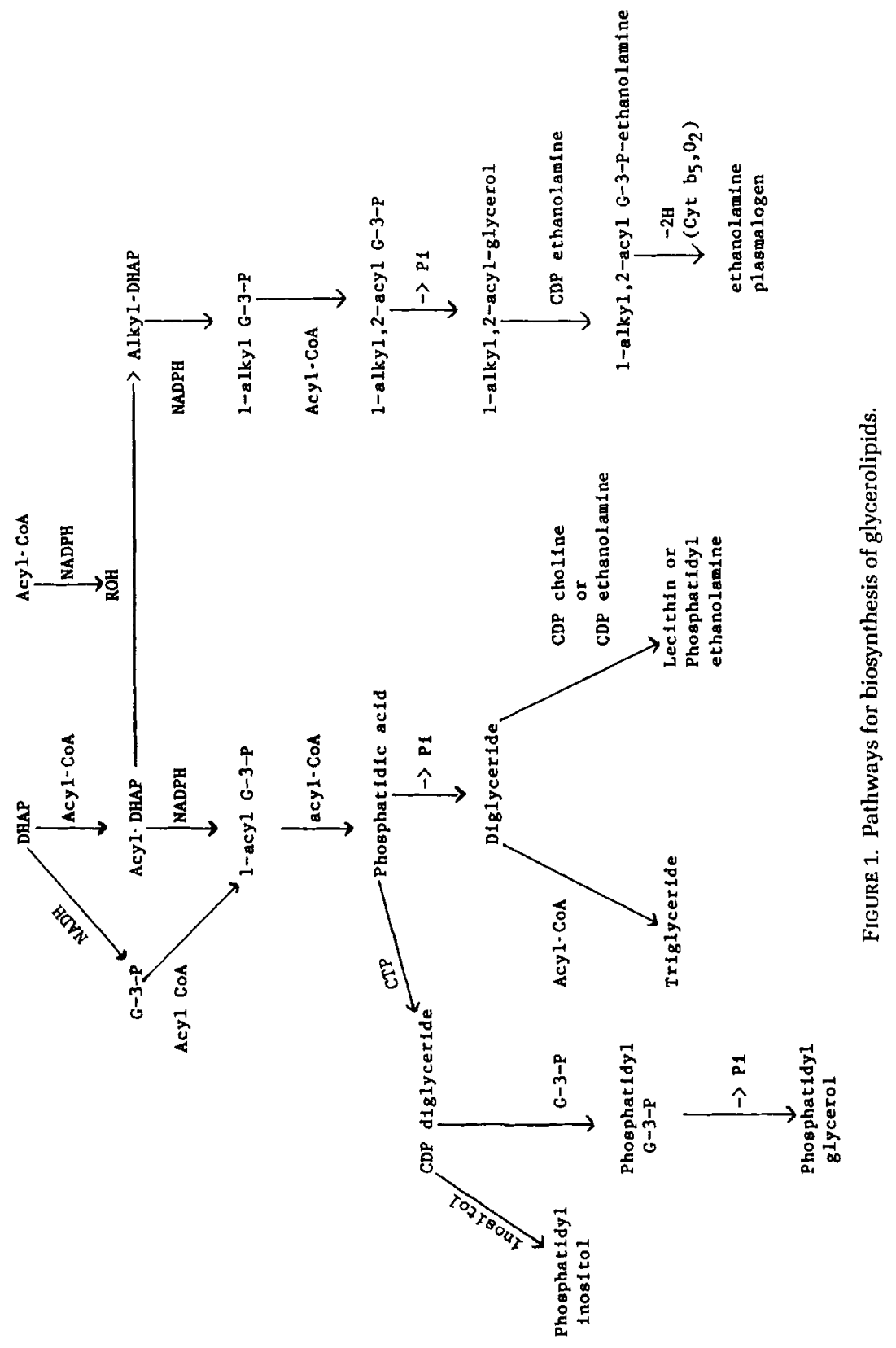


bimodal distribution; however, this enzyme has very low activity in rat liver making it difficult to quantify. In guinea pig liver this ether bond synthesizing enzyme has a higher activity and was shown to be predominantly localized in the light mitochondrial fraction (FIG. 2). ${ }^{18,19}$

The above results obtained from Triton-WR 1339-treated rats established that the key enzymes of the acyl-DHAP pathway are localized in peroxisomes. However, the in vivo use of the Triton WR 1339 to artificially lower the density of lysosomes poses a number of problems. The detergent has been shown to change the properties and enzyme profile of lysosomes. ${ }^{23}$ Though the detergent does not enter the peroxisomes, it has been reported that the activity of a number of

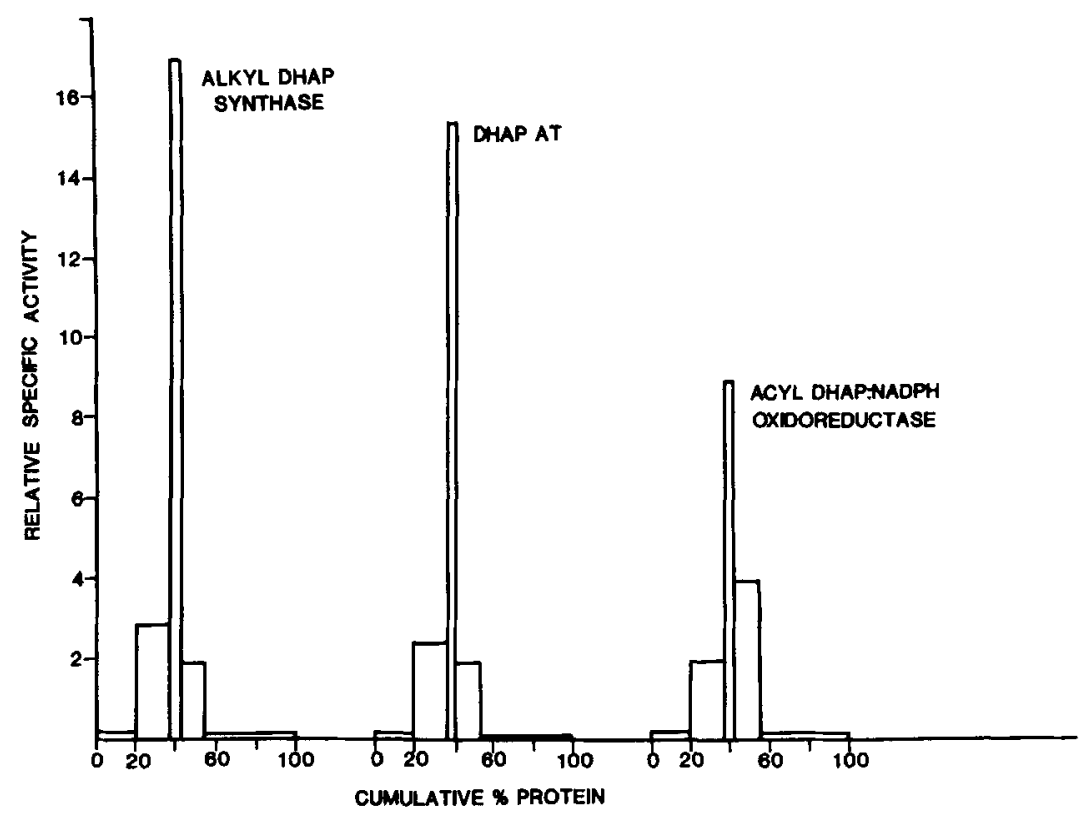

FIGURE 2. Distribution pattern of different acyl-DHAP pathway enzymes in the guinea pig liver subcellular fractions. Abscissa: fractions are represented by their relative protein content in the order in which they were isolated, i.e., from left to right nuclear fraction $[20 \%$ of the homogenate protein], mitochondrial fraction (15\% protein], light mitochondrial fraction [ $2.5 \%$ protein), microsomal fraction (13\% protein], and cytosol (49\% protein).

peroxisomal enzymes changes in the Triton-treated rats. ${ }^{24}$ Therefore, the enzyme distribution profile in these rats cannot be accepted as normal. Moreover, the Triton treatment to lower the density of lysosomes works only for liver, but not for other organs such as kidney. ${ }^{21}$ This method also may not work for the preparative isolation of peroxisomes from species other than rats. Our effort to purify peroxisomes from guinea pig liver using this detergent administration to animals was not successful, (Kroll \& Hajra, unpublished experiments) probably because of the high susceptibility of guinea pig liver peroxisomes to rupture by (endogenous) detergents.

For these reasons, a method was sought to isolate peroxisomes from normal 
animal organs uncontaminated with any other organelles. With the usual isolation method using differential centrifugation, the peroxisomes are heavily contaminated with lysosomes and mitochondria because of the similarity of their sizes. These organelles from normal rats cannot be separated from each other by density gradient centrifugation in sucrose solution because of their similarity in densities in this medium. ${ }^{21}$ However, a recent report by Wattiaux et al ${ }^{25}$ indicated that densities of peroxisomes, lysosomes, and mitochondria are different from one another in Metrizamide solution. These workers utilized this difference to develop a method for the purification of lysosomes and pointed out the feasibility of using the Metrizamide gradient to isolate peroxisomes. ${ }^{25}$ We recently adopted and modified this density gradient centrifugation method in Metrizamide medium to isolate peroxisomes which are found to be virtually free of mitochondria and lysosomes.

Basically, the method consisted of first isolating an enriched preparation of rat liver peroxisomes (i.e. "light mitochondrial" fraction), then subjecting the "LM" fraction to an isopycnic centrifugation in a linear Metrizamide density gradient medium ( $\rho=1.11$ to $1.33 ; 20-60 \%$ aqueous Metrizamide). For the density gradient centrifugation, a vertical rotor (Beckman VTi 50) at a speed of 40,000 $\mathrm{rpm}\left(\mathrm{g}_{\mathrm{av}}=130,000\right)$ for $60 \mathrm{~min}$ was used. FIGURE 3 shows the separation achieved between peroxisomes and other organelles employing this method. The peroxisomal marker enzymes (catalase and uric acid oxidase) are localized mostly in a band of mean density $\rho=1.23$ near the bottom of the gradient, well separated from that of mitochondrial marker enzyme (succinate-cyt. c reductase, $p=1.13$ ) and lysosomal marker enzyme (acid phosphatase, $\rho=1.12$ ). The microsomal marker enzyme (glc-6-Pase, $\rho=1.19$ ) is also separated from the peroxisomal band (FIG. 3). From these data it is evident that a fairly pure normal liver peroxisomal fraction can be isolated using this Metrizamide density gradient centrifugation technique. Following this procedure it was found that, as is the case with Triton-WR-1339 injected rats, DHAPAT in normal rat liver is mainly localized in peroxisomes (FIG. 3). A similar enzyme glycerol-3-P acyl transferase, which in microsomes has been postulated to be the same enzyme as DHAPAT ${ }^{28}$ is seen to be virtually absent from the peroxisomal fraction (FIG. 3). Acyl/alkyl DHAP: NADPH oxidoreductase was found to have a bimodal distribution in the sense that only part of this total liver enzyme activity is in peroxisomes and the rest is in microsomes (data not shown). Preliminary experiments indicate that rat liver alkyl-DHAP synthase has a similar bimodal distribution pattern between peroxisomes and microsomes.

A better separation of peroxisomes and microsomes can be achieved using a more shallow (20-50\% Metrizamide) density gradient medium during the centrifugal separation. This is illustrated in FIGURE 4 where normal guinea pig liver "light mitochondrial" fraction is subjected to centrifugal separation in the Metrizamide density gradient medium. As in the rat liver (FrG. 3), it is seen that the peroxisomes (marker enzyme catalase) are well separated from lysosomes (marker enzyme acid phosphatase) and mitochondria (marker enzyme succinatecytochrome $c$ reductase) in this gradient. Peroxisomes are also separated from microsomes (marker enzymes glucose-6-phosphatase and NADPH-cytochrome $c$ reductase) by this method (FIG. 4). When the distribution of acyl-DHAP pathway enzymes in this gradient was checked, it was found that, as in the rat liver, DHAPAT is localized mostly in peroxisomes (FIG. 4). Acyl-DHAP:NADPH oxidoreductase is present in both the peroxisomal and microsomal fractions (FIG. 4). However, in contrast to the rat, alkyl-DHAP synthase in the guinea pig liver is present mostly in the peroxisomal fraction (FIG. 4). These results established that 
Hajra \& Bishop: Glycerolipid Biosynthesis

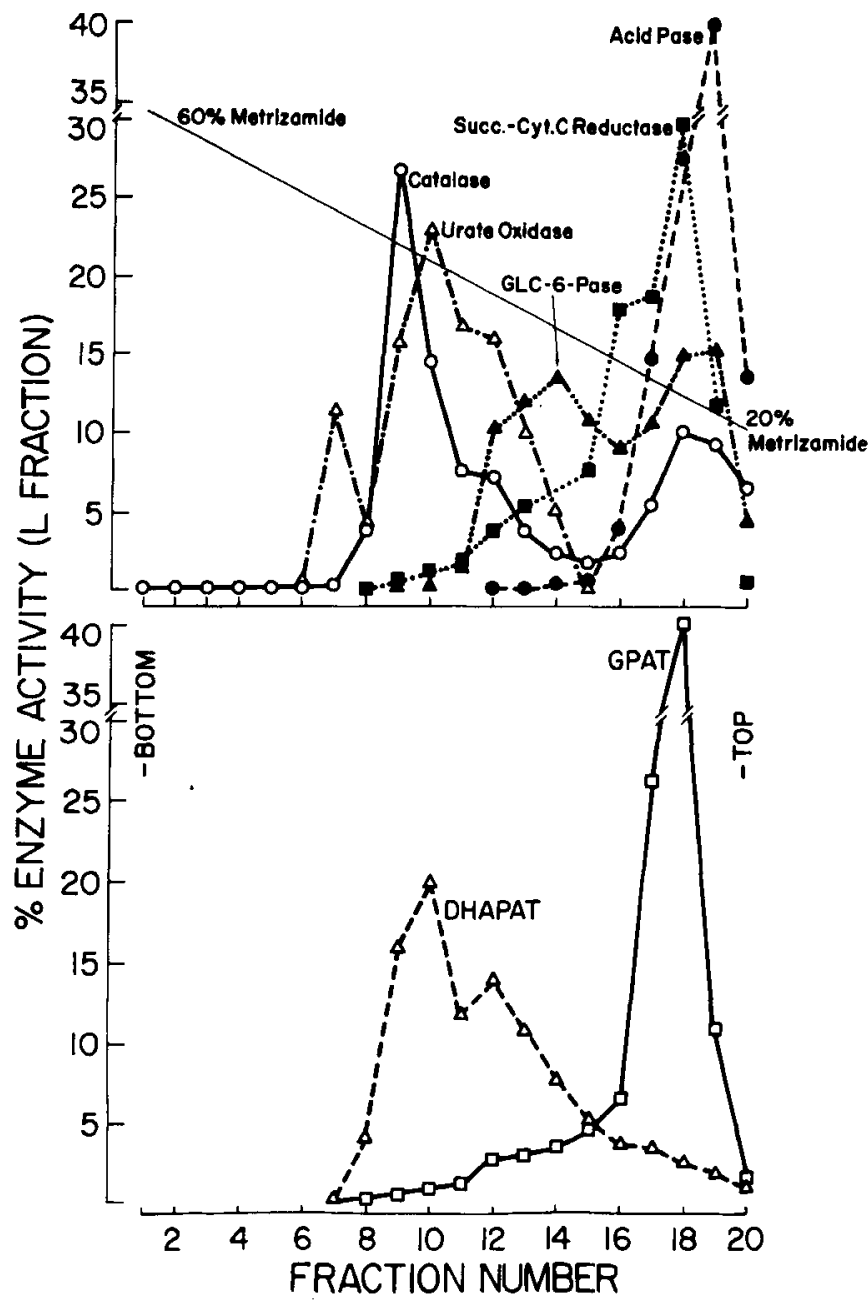

FIGURE 3. Distribution pattern of different enzymes after centrifugation in a linear Metrizamide density gradient $(20-60 \%$ Metrizamide density $1.11 \mathrm{~g} / \mathrm{ml}$ to $1.33 \mathrm{~g} / \mathrm{ml}$ ) of a rat liver light mitochondrial fraction. The rat liver fraction, suspended in $0.25 \mathrm{M}$ sucrose was layered on the top of the gradient tube, which was spun in a vertical rotor (Beckman VTi 50) at $40,000 \mathrm{rpm}\left(130,000 \mathrm{~g}_{\mathrm{av}}\right)$ for $60 \mathrm{~min}$. Following centrifugation, $2 \mathrm{ml}$ of fractions were collected from the bottom, and each fraction was assayed for different enzyme activity as shown in the figure. The enzyme activity is expressed as percent of total activity recovered from the gradient.

the acyl-DHAP pathway enzymes of normal guinea pig liver are localized in peroxisomes.

This preparative method of isolation of fairly pure peroxisomes will be useful in mapping the distribution of different enzymes in peroxisomes. Our main goal is to firmly establish the nature of all the lipid-metabolizing enzymes present in 


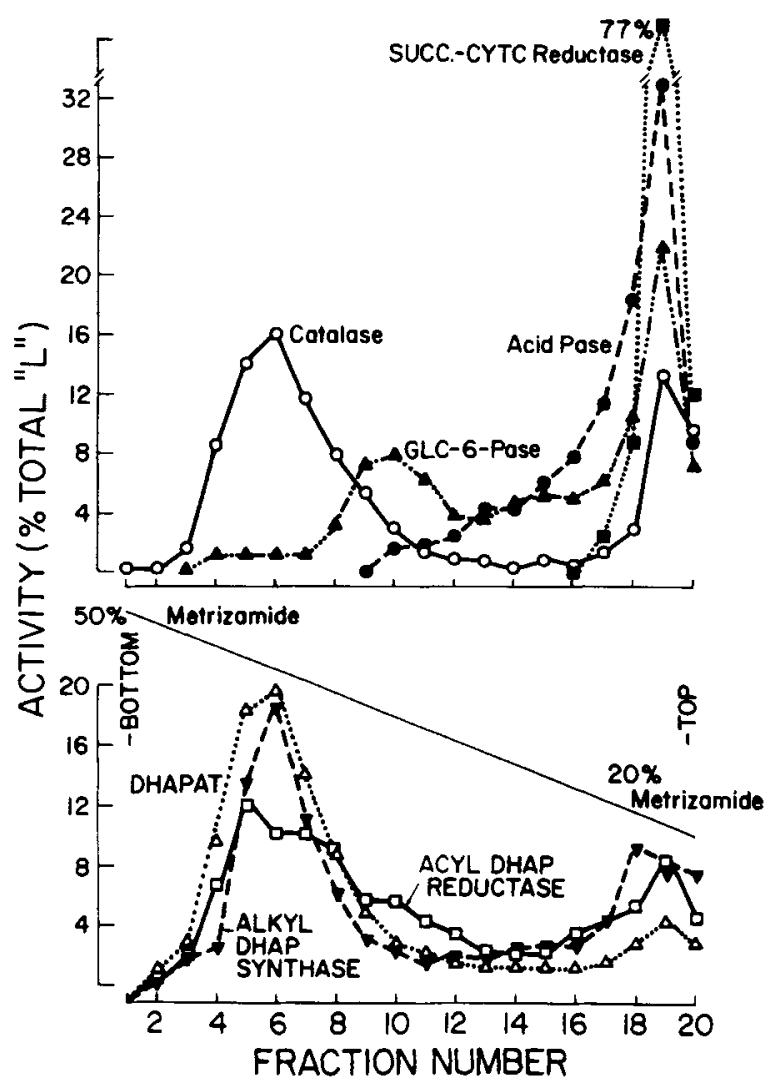

FIGURE 4. Distribution pattern of different enzymes of guinea pig liver light mitochondrial fraction after centrifugation in a Metrizamide density gradient (20-50\% Metrimazide $1.11 \mathrm{~g} / \mathrm{ml}$ to $1.28 \mathrm{~g} / \mathrm{ml}$ ). The method is similar to that described under FIGURE 3 .

peroxisomes, from which a possible role of peroxisomes in cellular lipid metabolism can be determined. Preliminary experiments show that the key enzymes which catalyze the biosynthesis of triglyceride or membrane phosphoglycerides, such as diacylglycerol acyl transferase (EC 2.3.1.20) choline phosphotransferase (EC 2.7.8.2), CDP diglyceride:inositol phosphatidyl transferase (EC 2.7.8.11], etc., are virtually absent in the isolated liver peroxisomes.

The presence of acyl-DHAP pathway enzymes in tissues other than liver and kidney raises the interesting question about the possible site of localization of these enzymes in such tissues. Strictly speaking, peroxisomes as defined, i.e., "cytoplasmic organelles characterized by the association of catalase and one or more $\mathrm{H}_{2} \mathrm{O}_{2}$-producing oxidases," ${ }^{27}$ are present only in liver and kidney. The particles in liver and kidney are comparable in size $[0.5 \mu \mathrm{m}-1.0 \mu \mathrm{m})$ to that of mitochondria and lysosomes ${ }^{28}{ }^{2}$ However, it has been shown that smaller catalasecontaining particles $(0.1 \mu \mathrm{m}-0.25 \mu \mathrm{m}$ diameter $)$, which have been termed "microperoxisomes" by Novikoff and coworkers, ${ }^{26.29}$ are ubiquitous in animal tissues. It is possible that in tissues other than liver and kidney, acyl-DHAP 
pathway enzymes are localized in microperoxisomes. This would explain the previously discussed anomalous distribution pattern of the acyl-DHAP pathway enzymes obtained in different tissues, i.e. present in crude mitochondrial fraction from liver and kidney, but enriched in microsomal fractions of other organs. ${ }^{6.16}$ On differential centrifugation, the larger peroxisomes of liver and kidney tend to sediment with the mitochondria whereas the smaller microperoxisomes sediment down in the microsomal fraction. ${ }^{28}$ Though microperixosomes are widespread in different tissues, a general method for preparative isolation of microperoxisomes has not yet been developed. We have attempted to purify microperoxisomes from young rat brain. Holtzman and coworkers ${ }^{30}$ have shown by cytochemical techniques that numerous microperoxisomes are present in developing rat brain in cytoplasm and in presynaptic terminals. A method was developed in our laboratory to concentrate catalase-containing particles from 12-day-old rat brain particulate fraction. This fraction was repeatedly frozen and thawed to release microperoxisomes from synaptosomes, then separated using a Metrizamide-saline density gradient centrifugation. The results are shown in FIGURE 5, where it is seen that catalase-containing particles are partially separated from microsomes (marker enzyme estrone sulfatase and also NADPHcytochrome $c$ reductase) after density gradient centrifugation. The distribution pattern of two of the acyl-DHAP pathway enzymes, i.e., DHAPAT and alkylDHAP reductase, are similar to that of catalase, indicating that in brain these enzymes are localized in microperoxisomes. As in liver, a portion of the reductase activity seemed to be associated with microsomes. Our recent findings also indicate that two other enzymes of the acyl DHAP pathway, i.e., alkyl-DHAP synthase and acyl-CoA:NADPH oxidoreductase (long-chain alcohol-forming) ${ }^{11}$ are also localized in brain microperoxisomes. It may be a general phenomenon

Figure 5. Distribution pattern of different enzymes of young (12-dayold] rat brain cerebrum particulate fraction after centrifugation in a $0.9 \%$ saline-Metrizamide gradient [8.5-36.5\% Metrizamide]. The total particulate fraction of cerebrum (sedimenting between $5 \times 10^{3} \mathrm{~g}$-min and $8.4 \times 10^{6} \mathrm{~g}$-min] was frozen $\left(-70^{\circ}\right)$ and thawed $\left(37^{\circ} \mathrm{C}\right)$ seven times in $0.32 \mathrm{M}$ sucrose and then layered on the gradient and centrifuged at $40,000 \mathrm{rpm}\left(\mathrm{g}_{\mathrm{av}}=201,000\right)$ in a Beckman SW40.1 rotor for $30 \mathrm{~min}$. From the bottom, $0.75 \mathrm{ml}$ of fractions were collected and different enzyme activities (as shown) were measured in each fraction.

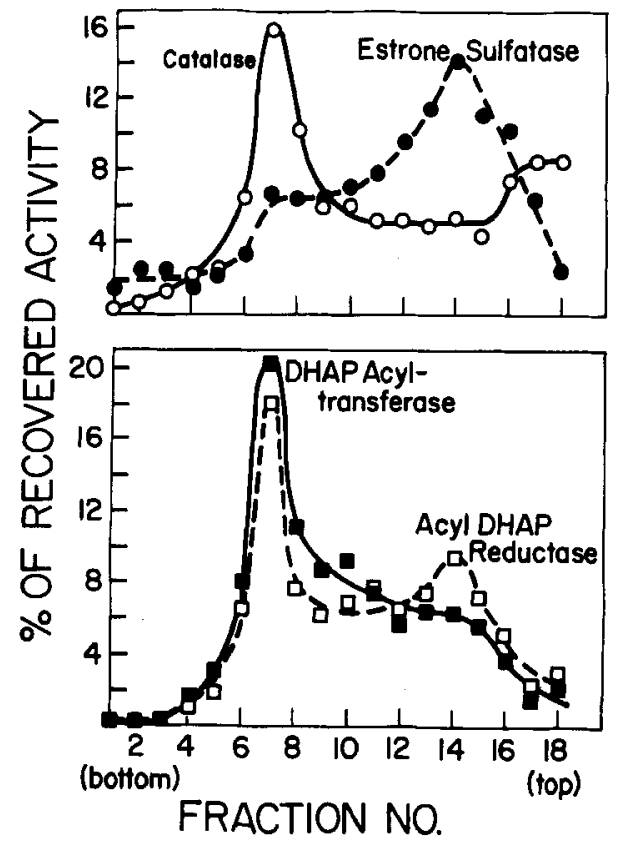


that in organs other than liver and kidney the acyl-DHAP pathway enzymes are localized in microperoxisomes.

The possible role of acyl-DHAP pathway enzymes in peroxisomes is still not clear. The apparent lack of other lipid biosynthesizing enzymes in peroxisomes raises the possibility that the acyl-DHAP pathway enzymes may have roles other than for the biosynthesis of glycerolipids. In recent years, peroxisomes have been shown to contain an active long-chain acyl-CoA $\beta$-oxidation enzyme system. ${ }^{31}$ However, an efficient transport system of long-chain acyl-CoA's across the peroxisomal membrane has not yet been reported. Peroxisomes contain shortchain (up to $\mathrm{C}_{8}$ ) but no long-chain carnitine acyl transferase ${ }^{32}$ It is possible that acyl-DHAP, like acyl carnitines in mitochondria, ${ }^{33}$ may participate in transporting long-chain acyl groups across peroxisomal membranes. Similar to that of the biosynthesis of acyl-carnitines, the reaction catalyzed by DHAPAT has been shown to be reversible so that acyl-CoA is formed when acyl-DHAP is incubated with $\mathrm{CoA}$ in the presence of DHAPAT ${ }^{34}$ DHAPAT is probably localized on the inside face of peroxisomal membrane as most of it was shown to be resistant to trypsin digestion in the absence of any detergent..$^{35}$ However, a part of liver DHAPAT $(20-30 \%)$ is labile towards trypsin (Bishop, Salem \& Hajra, this volume). Though it is not yet established whether this portion of proteolytic enzyme-labile DHAPAT is due to the presence of damaged peroxisomes, it is possible that a portion of DHAPAT is also localized on the outer side of the peroxisomal membrane. A scheme may be proposed with the analogy to the acyl carnitine-mediated transport of the acyl group in mitochondria, where acylDHAP is formed at the outside of peroxisomes, i.e.:

$$
\text { acyl-CoA }+ \text { DHAP } \rightarrow \text { acyl-DHAP }+ \text { CoA }
$$

Acyl DHAP is then transported across the membrane to the inside of peroxisomes where it reacts with $\mathrm{CoA}$ to regenerate acyl-CoA:

$$
\text { acyl-DHAP + CoA } \rightarrow \text { acyl-CoA + DHAP }
$$

This latter reaction may be facilitated by the removal of DHAP when it is reduced to G-3-P inside the peroxisomes by NADH, catalyzed by the peroxisomal glycerol-3-phosphate dehydrogenase ${ }^{36}$ (FIG. 6). This scheme also provides a mechanism by which the reducing equivalent from NADH could be carried across peroxisomal and mitochondrial membranes via glycerol-3-phosphate (glycerophosphate shuttle), which is oxidized in mitochondria to DHAP (FIG, 6). The reducing equivalent of NADPH generated inside the peroxisomes may also be carried from peroxisomes to mitochondria via 1-acyl-G-3-P, as shown in FIG. 6. It should be emphasized here that the scheme put forward in FIG. 6 is purely hypothetical as there is no experimental evidence to support this model.

The above scheme does not explain the role of all of the lipid-metabolizing enzymes (e.g. alkyl-DHAP synthase) present in peroxisomes. Peroxisomes may be involved in cellular membrane lipid biogenesis. From a number of other observations, peroxisomes have also been postulated to have a role in lipid metabolism. For example, Novikoff and coworkers have shown that in a number of histological preparations peroxisomes are always closely associated with lipid droplets. ${ }^{37.38}$ Tissues that are very active in lipid metabolism such as intestinal mucosa, liver, adrenal gland, testis, brown adipose tissue, etc. have been shown to contain a large number of microperoxisomes. ${ }^{28}$ Recently. Arnold and Holtzman $^{30}$ demonstrated that microperoxisomes are numerous in developing rat brain during the period of active myelination and synaptogenesis. The most convincing 


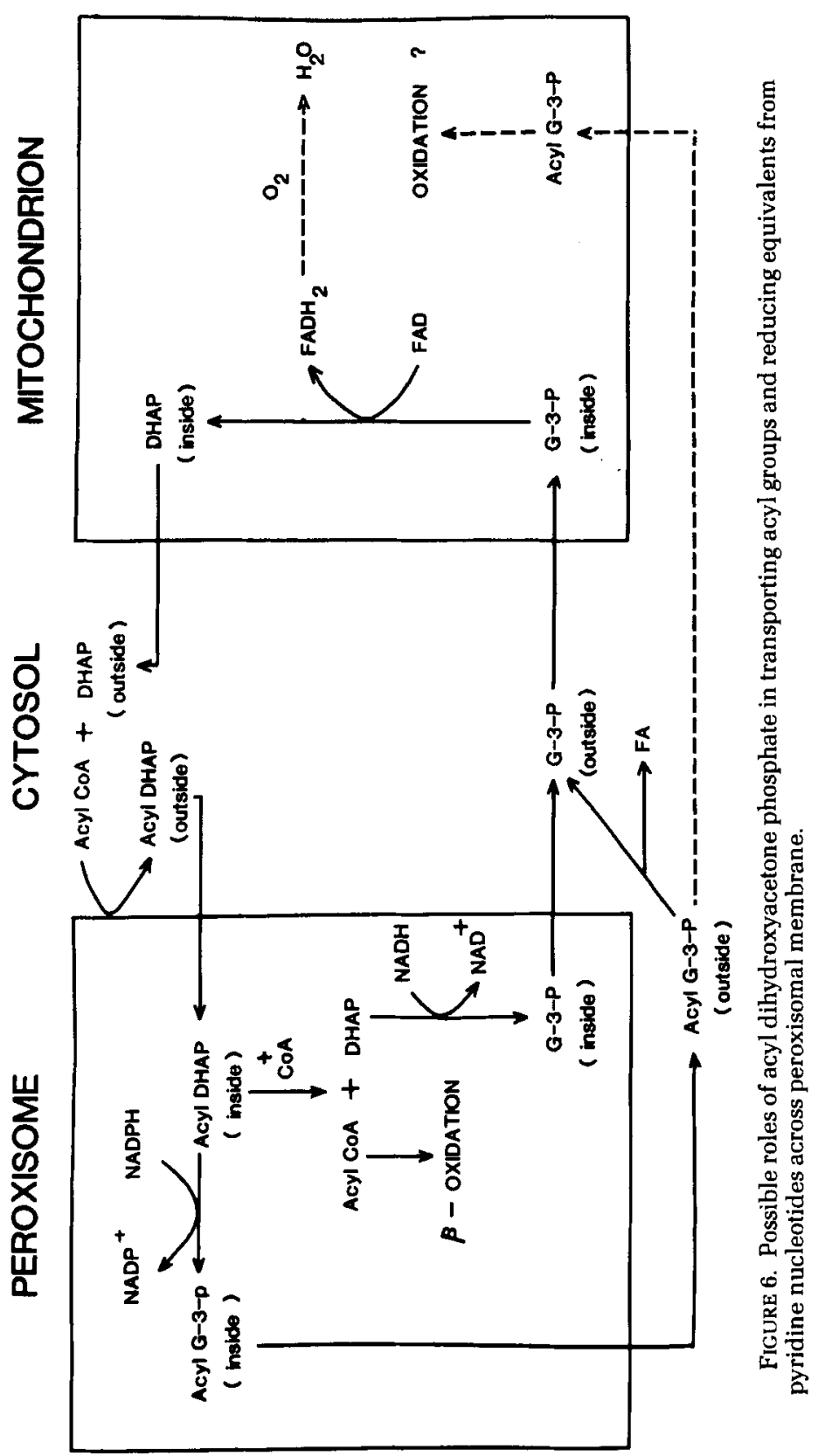


evidence for the role of peroxisomes in lipid metabolism is the finding that a number of hypolipidemic agents induce a proliferation of peroxisomes in liver. ${ }^{28}$ This was first observed by Hess et $a l^{39}$ with the drug Clofibrate (ethyl $p$ chlorophenoxy isobutyric acid), which is clinically used to lower serum triglyceride and cholesterol levels. Following this report a number of other hypolipidemic agents (Clofenapate, Tibric acid, WY 14643, etc.), some of which are structurally unrelated to clofibrate, ${ }^{40.41}$ were shown to induce a similar proliferation of peroxisomes in rat liver. This phenomenon has been extensively studied in the last few years by different workers, and it has been reported that this proliferation of peroxisomes by drugs is generally accompanied by an increase in liver weight (hepatomegaly) ${ }^{28}$ and also by an increase in the peroxisomal enzymes of fatty acid $\beta$-oxidation cycle ${ }^{42}$ and in the peroxisomal carnitine acetyl transferase. ${ }^{43}$ We have previously reported that chronic administration of clofibrate to rats resulted in an increase in the specific activity and total activity of liver DHAPAT and G-3-PAT. ${ }^{44}$ Recently we found a similar increase in the activity of these lipid metabolizing enzymes with two other hypolipedemic drugs, Bezafibrate (Boehringer-Mannheim) and Wy-14,643 (Wyeth Lab.) (Aquilina \& Hajra, unpublished works). (However, Gemfibrozil (Warner-Lambert), another hypolipidemic drug, or phenobarbital, a microsome proliferator, did not elicit such increases in liver DHAPAT and GPAT.] This paradoxical effect, i.e., increase in lipid biosynthetic enzymes by hypolipidemic drugs, may be explained as a compensatory mechanism because these drugs have been shown to inhibit these enzymes in vitro. ${ }^{45}$ This increase in DHAPAT and GPAT may not be specifically related to the hypolipidemic action of these drugs, however, but may be associated with the proliferation of liver tissue induced by these drugs ${ }^{28}$ in that this hepatomegaly would entail an increased rate of membrane lipid biosynthesis.

All these above findings point towards a strong association of peroxisomes with cellular lipid metabolism. Peroxisomes may supply lipid precursors such as acyl-DHAP or alkyl-DHAP or lysophosphatidic acid to endoplasmic reticulum for lipid synthesis and thus may provide some kind of substrate level control system for cellular membrane biogenesis. However, our present knowledge about the enzymes in peroxisomes permits only speculative proposals to be advanced regarding their role in the metabolic activity of the cell.

\section{REFERENCES}

1. HajRA, A. K. 1977. Biochem. Soc. Trans. 5: 34-36.

2. VAN DEN BOSCH, H. 1974. Ann. Rev. Biochem. 43: 243-277.

3. HajRA, A. K. \& B. W. AGRanOFF. 1968. J. Biol. Chem. 243: 1617-1622.

4. HajRA, A. K. 1968. J. Biol. Chem. 243: 3458-3465.

5. HajRA, A. K. \& B. W. AGRANOFF. 1968. J. Biol. Chem. 243: 3542-3543.

6. LABELLE, E. F., JR. \& A. K. HajRA. 1972. J. Biol. Chem. 247: 5825-5834.

7. Kennedy, E. P. 1961. Fed. Proc. 20: 934-940.

8. HajRA, A. K. 1970. Biochem. Biophys. Res. Commun. 39: 1037-1044.

9. WYKLE, R. L. \& F. SNYDER. 1976. In The Enzymes of Biological Membranes. A. MARTONOSI, Ed. Vol. 2: 87-117. Plenum. New York.

10. FriedBerg, S. J. \& A. HeIfETZ. 1975. Biochemistry 14: 570-574.

11. Bishop, J. E. \& A. K. HAJRA. 1981. J. Biol. Chem. 256: 9542-9550.

12. Paltauf, F. \& A. Holosek. 1973. J. Biol. Chem. 248: 1609-1615.

13. Pollock, R. J., A. K. HajRa \& B. W. Agranoff. 1976. J. Biol. Chem. 251: 5149-5154.

14. MANning, R. \& D. N. BrindLey. 1972. Biochem. J. 130: 1003-1012. 
15. Mason, R. J. 1978. J. Biol. Chem. 253: 3367-3370.

16. LaBElle, E. F., JR. \& A. K. HAJRA. 1972. J. Biol. Chem. 247: 5835-5841.

17. Davis, P. A. \& A. K. HajRA. 1981. Arch. Biochem. Biophys. 211: 20-29.

18. DeDuve, C., B. C. Pressman, R. Gianetto, R. Wattlaux \& F. Appelmans. 1955. Biochem. J. 60: 604-617.

19. JONES, C. L. \& A. K. HAJRA. 1977. Biochem. Biophys. Res. Commun. 76: 1138-1143.

20. HajRA, A. K., C. L. Burke \& C. L. Jones. 1979. J. Biol. Chem. 254: 10896-10900.

21. Leighton, F., B. Poole, H. Beaufay, P. Baudhuin, J. W. Coffey, S. Fowlers \& C. DE Duve. 1968. J. Cell. Biol. 37: 482-513.

22. HajRa, A. K., C. L. Jones \& P. A. Davis. 1978. In Enzymes of Lipid Metabolism. S. GATT, L. Freysz \& P. MANDEL, Eds. p:369-378. Plenum. New York.

23. DAVIES, M. 1973. Biochem. J. 136: 57-65.

24. Ishil, H., T. Suga, H. Hayashi \& S. Ninobe. 1979. Biochim. Biophys. Acta 582: 213220.

25. Wattiaux, R., S. WattiauX-De Connick, M. Ronveaux-Dupal \& F. Dubois. 1978. J. Cell. Biol. 78: 349-368.

26. Schlossman, D. M. \& R. M. Bell. 1977. Arch. Biochem. Biophys. 182: 732-742.

27. DE Duve, C. \& P. Baudhuin. 1966. Physiol. Rev. 46: 323-357.

28. Böck, P., R. Kramar, \& M. Pavelka. 1980. Peroxisomes and Related Particles in Animal Tissues. Springer-Verlag. New York.

29. Novikoff, A. B. \& P. M. Novikoff. 1973. J. Histochem. Cytochem. 21: 963-966.

30. ARnOld, G. \& E. Holtzman. 1978. Brain Res. 155: 1-17.

31. Lazarow, P. B. 1978. J. Biol. Chem. 253: 1522-1528.

32. Markwell, M. A. K., E. J. McGroarty, L. L. Bieber \& N. E. Tolbert, 1973. J. Biol. Chem. 248: 3426-3432.

33. Bressler, R. 1970. In Lipid Metabolism. S. Wakil, Ed.: 49-77. Academic Press. New York.

34. HAJRA, A. K. 1974. Biochem. Biophys. Res. Commun. 57: 668-674.

35. JONES, C. L. \& A. K. HAJRA. 1980. J. Biol. Chem. 255: 8289-8295.

36. Gee, R., E. MCGroarty, B. Hsieh, D. M. Wied \& N. E. Tolbert. 1974. Arch. Biochim. Biophys. 161: $187-193$.

37. NovikofF, A. B. \& W. B. ShiN. 1964. J. Micros. 3: 187-206.

38. Novikoff, A. B., P. M. Novikoff, O. M. Rosen \& C. S. Rubin. 1980. J. Cell Biol. 87: $180-196$.

39. Hess, R., W. Staubl \& W. RiEss. 1965. Nature 208: 856-858.

40. REDDY, J. K. \& T. KRISHNAKANTHA. 1975. Science 190: 787-789.

41. MoOdy, D. E. \& J, K. Reddy. 1976. J. Cell Biol. 71: 768-780.

42. LAZAROW, P. B. 1977. Science 197: 580-581.

43. Moody, D. E. \& J. K. Reddy. 1974. Res. Commun. Chem. Pathol. Pharmacol. 9: 501510.

44. BURKE, C. L. \& A. K. HAJRA. 1980. Biochem. Internat. 1: 312-318.

45. HajRA, A. K. 1981. Fed. Proc. 40: 1630.

\section{Discussion OF THE PAPER}

F. Leighton (Universidad Católica de Chile, Santiago): It has been observed that the activity of some enzymes from the plasmalogen biosynthetic pathway is enhanced by clofibrate administration: dihydroxyacetone phosphate acyl transferase (Burke \& Hajra, Biochem. Int. 1: 312-318, 1980) and dihydroxyacetone phosphate alkyl transferase (Shinojima, Ono \& Imai, shown at the XI Int. Congress Biochem. Toronto, 1979). Using this information and considering the possibility that one of these enzymes might be rate limiting for plasmalogen biosynthesis, with $\mathrm{E}$. Brandan we have explored in our laboratory the effect of 
ciprofibrate-a clofibrate analogue-on the plasmalogen content of various rat tissues. After one to two weeks of administration $(30 \mathrm{mg} / \mathrm{kg}$ food) the drug had no effect on the phosphatidyl choline, P.C.; phosphatidyl ethanolamine, P.E.; phosphatidal choline, Pl.C; or phosphatidal ethanolamine, Pl.E., content in liver, brain and harderian gland. In plasma, P.C. decreased from $61.7 \pm 2.3$ to $54.4 \pm 0.9$ percent of total lipid phosphorus. In red cell membranes, P.E. increased from $8.4 \pm 0.8$ to $14.9+0.9$ and Pl.E. from $9.2 \pm 0.6$ to $16.4 \pm 1.8$. The increase detected in red cell membrane plasmalogens, Pl.E., is paralleled by the increase in P.E., suggesting that ciprofibrate acts at a different level, presumably common to both forms of the ethanolamine phospholipid, and not on the enzymes mentioned. The change in plasma P.C. content constitutes another effect of the drug apparently also independent from the changes described for the plasmalogen biosynthesis enzymes.

A. K. HajRa (University of Michigan, Ann Arbor): We know that clofibrate increases not only dihydroxyacetone phosphate acyl transferase but also glycerol phosphate transferase. I suggest that this has nothing to do with the hypolipidemic action of these drugs but rather that the stimulation of lipid enzymes accounts for hepatomegaly in that there is an increased synthesis of membranes in liver cells.

E. Holtzman (Columbia University, New York): Working with the developing brain, I have shown a very interesting localization of microperoxisomes in close association with myelin sheets in the oligodendrocytes during the stages of myelination. At this stage the brain peroxisomes were made. Details can be seen in my poster presentation in this volume. 